\title{
Intersection patterns of convex sets via simplicial complexes, a survey
}

\author{
Martin Tancer*
}

October 25, 2011

\begin{abstract}
Keywords: convex set, intersection pattern, simplicial complex, $d$ representability, $d$-collapsibility, Helly-type theorem
\end{abstract}

MSC2010 Classification: primary 52A35, secondary 05E45, 52A20

\begin{abstract}
The task of this survey is to present various results on intersection patterns of convex sets. One of main tools for studying intersection patterns is a point of view via simplicial complexes. We recall the definitions of so called $d$-representable, $d$-collapsible and $d$-Leray simplicial complexes which are very useful for this study. We study the differences among these notions and we also focus on computational complexity for recognizing them. A list of Helly-type theorems is presented in the survey and it is also discussed how (important) role play the above mentioned notions for the theorems. We also consider intersection patterns of good covers which generalize collections of convex sets (the sets may be 'curvy'; however their intersections cannot be too complicated). We mainly focus on new results.
\end{abstract}

\section{Introduction}

An important branch of combinatorial geometry regards studying intersection patterns of convex sets. Research in this area was initiated by a theorem of Helly [Hel23] which can be formulated as follows: If $C_{1}, \ldots, C_{n}$ are convex sets in $\mathbb{R}^{d}, n \geq d+1$, and any collection of $d+1$ sets among $C_{1}, \ldots, C_{n}$ has

\footnotetext{
${ }^{*}$ Department of Applied Mathematics and Institute for Theoretical Computer Science (supported by project 1M0545 of The Ministry of Education of the Czech Republic), Faculty of Mathematics and Physics, Charles University, Malostranské nám. 25, 11800 Prague, Czech Republic. Partially supperted by project GAUK 421511.
} 
a nonempty intersection, then all the sets have a common point. We will focus on results of similar spirit; however, we have to set up some notation first.

\subsection{Simplicial complexes}

First we recall simplicial complexes which provide a convenient language for studying intersection patterns of convex sets. We assume that the reader is familiar with simplicial complexes, we only briefly mention the basics. For further details the reader is referred to the books like [Hat01, Mat03, Mun84.

We deal with finite abstract simplicial complexes, i.e., collections $\mathrm{K}$ of subsets of a finite set $X$ such that if $\alpha \in \mathrm{K}$ and $\beta \subset \alpha$, then $\beta \in \mathrm{K}$. Elements of $\mathrm{K}$ are faces of $\mathrm{K}$. The dimension of a face $\alpha \in \mathrm{K}$ is defined as $|\alpha|-1$; $i$-dimensional faces for $i \in\{0,1,2\}$ are vertices, edges and triangles respectively. The dimension of a simplicial complex is the maximum of dimensions of its faces. Graphs coincide with 1-dimensional simplicial complexes. If $V^{\prime}$ is a subset of vertices of $\mathrm{K}$ then the induced subcomplex $\mathrm{K}\left[V^{\prime}\right]$ is a complex of faces $\alpha \in \mathrm{K}$ such that $\alpha \subseteq V^{\prime}$. We use the notation $\mathrm{L} \leq \mathrm{K}$ for pointing out that $\mathrm{L}$ is an induced subcomplex of $\mathrm{K}$. An $m$-skeleton of $\mathrm{K}$ is a simplicial complex consisting of faces of $\mathrm{K}$ of dimension at most $m$. We denote it by $\mathrm{K}^{(m)}$. The $m$-dimensional full simplex, $\Delta_{m}$, is a simplicial complex with the vertex set $\{1, \ldots, m+1\}$ and all possible faces. Let $Y$ be a set of affinely independent points in $\mathbb{R}^{|X|-1}$ such that there is a bijection $f: X \rightarrow Y$. The geometric realization of $\mathrm{K}$, denoted by $|\mathrm{K}|$, is the topological space $\bigcup\{\operatorname{conv} f(\alpha): \alpha \in \mathrm{K}\}$, where conv denotes the convex hull.

\section{$1.2 \quad d$-representable complexes}

Let $\mathcal{C}$ be a collection of some subsets of a given set $X$. The nerve of $\mathcal{C}$, denoted by $\mathrm{N}(\mathcal{C})$, is a simplicial complex whose vertices are the sets in $\mathcal{C}$ and whose faces are subcollections $\left\{C_{1}, \ldots, C_{k}\right\} \subseteq \mathcal{C}$ such that the intersection $C_{1} \cap \cdots \cap C_{k}$ is nonempty. The notion of nerve is designed to record the 'intersection pattern' of the sets in $\mathcal{C}$.

A simplicial complex $\mathrm{K}$ is $d$-representable if it is isomorphic to the nerve of a finite collection of convex sets in $\mathbb{R}^{d}$. Such a collection of convex sets is called a $d$-representation for $\mathrm{K}$. $d$-representable simplicial complexes are the central objects of our study in this survey. As it was mentioned above, they exactly record all possible intersection patterns of finite collections of convex sets in $\mathbb{R}^{d}$. 


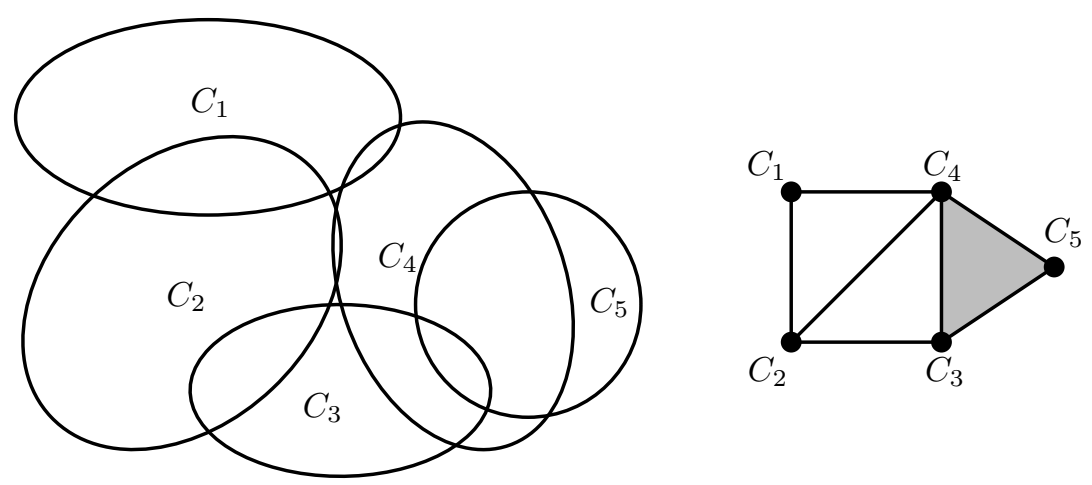

Figure 1: A 2-representable complex and its nerve.

Using the notion of $d$-representability, the Helly theorem can be reformulated as follows: If a $d$-representable complex on at least $d+1$ vertices contains all possible $d$-faces, then it is already a full simplex. This statement is, via induction, equivalent with the following: $d$-representable simplicial complex does not contain an induced $k$-dimensional simplicial hole for $k \geq d$, i.e., a complex isomorphic to $\Delta_{k+1}^{(k)}$. (Note that "hole" refers here to a hole in a certain topological space, and in particular this notion is different from $k$-hole in the context of Horton sets. The dimension of the hole refers to the dimension of the boundary rather than to the dimension of the missing part. We also remark that a geometric representation of $k$-dimensional simplicial hole is homeomorphic to the $k$-sphere $S^{k}$; and it is the simplest way to obtain the $k$-sphere as a simplicial complex.) The Helly number of a simplicial complex $\mathrm{K}$ is the total number of vertices of the largest simplicial hole in $\mathrm{K}$ (i.e., the dimension of the hole plus 2). Another reformulation of the Helly theorem thus states that the Helly number of a $d$-representable complex is at most $d+1$.

Example 1.1. Figure 1 shows a collection $\mathcal{C}=\left\{C_{1}, C_{2}, C_{3}, C_{4}, C_{5}\right\}$ of convex sets (on left) and their nerve (on right). In other words the simplicial complex on right is 2-representable and $\mathcal{C}$ is a 2-representation of it. The Helly number of this collection equals 3 .

\subsection{What is in the survey?}

The task of the survey is to give an overview of a recent developments on the study of intersection patterns of finite collections of convex sets. We 
are mainly focusing on the description of intersection patterns via simplicial complexes. Apart from geometrical 'ad hoc' arguments there are two other important approaches we are going to discuss. First of them is combinatorial and regards $d$-collapsibility. The second one is topological and regards the Leray number of a simplicial complex. These two approaches are mainly discussed in the following two sections. Algorithmic aspects of recognition of intersection patterns are briefly discussed in section 4. In section 5 we mention some properties of good covers as a natural generalization of collections of convex sets. Finally, section 6 contains a list of theorems on intersection patterns. This list is at the end of the survey in order that all the necessary terminology is already built up. However, many of the results in section 6 can be understand without a detailed study of the previous sections.

Since our task is to cover only a very selected part of convex geometry, we refer the reader to other sources regarding the related areas. In particular we refer to Mat02 and the references therein for extended basic overview on discrete convex geometry including Radon, Carathéodory, and Tverberg type theorems; we also refer to [Eck93] for another point of view on the area; and to GPW93 for results on transversals to convex sets. We also do not focus on the theory of $f$-vectors. For a reader interested in $f$-vectors we refer to [BB97 or to Kal02 for a useful method for investigating $f$-vectors (and related also to other branches mentioned here).

\section{$2 d$-collapsible and $d$-Leray complexes}

There are two other important classes of simplicial complexes related to the $d$-representable ones. Informally, a simplicial complex is $d$-collapsible if it can be vanished by removing faces of dimension at most $d-1$ which are contained in a single maximal face; a simplicial complex is $d$-Leray if its induced subcomplexes do not contain, homologically, holes of dimension $d$ or more.

Wegner Weg75 proved that $d$-representable simplicial complexes are $d$-collapsible and also that $d$-collapsible complexes are $d$-Leray.

Now we precisely define $d$-collapsible complexes and then $d$-Leray complexes.

Let $\mathrm{K}$ be a simplicial complex. Let $T$ be the collection of inclusion-wise maximal faces of $\mathrm{K}$. A face $\sigma$ is $d$-collapsible if there is only one face $\tau \in T$ containing $\sigma$ (possibly $\sigma=\tau$ ), and moreover $\operatorname{dim} \sigma \leq d-1$. The simplicial complex

$$
\mathrm{K}^{\prime}:=\mathrm{K} \backslash\{\eta \in \mathrm{K}: \eta \supseteq \sigma\}
$$




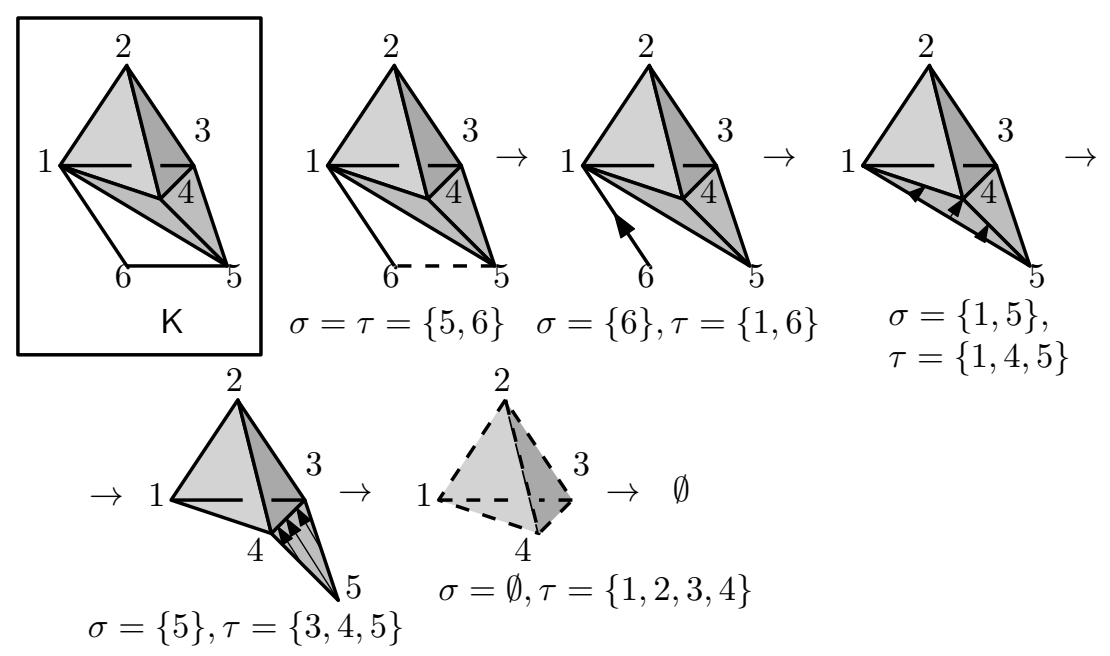

Figure 2: A 2-collapsing of a simplicial complex.

is an elementary $d$-collapse of $\mathrm{K}$. For such a situation, we use the notation $\mathrm{K} \rightarrow \mathrm{K}^{\prime}$. A simplicial complex is d-collapsible if there is a sequence,

$$
\mathrm{K} \rightarrow \mathrm{K}_{1} \rightarrow \mathrm{K}_{2} \rightarrow \cdots \rightarrow \emptyset
$$

of elementary $d$-collapses ending with an empty complex.

Example 2.1. A simplicial complex $\mathrm{K}$ consisting of a full tetrahedron, two full triangles and one hollow triangle in Figure 2 is 2-collapsible. For a proof there is a 2-collapsing of $\mathrm{K}$ drawn on the picture. In every step the faces $\sigma$ and $\tau$ are indicated.

A simplicial $\mathrm{K}$ complex is $d$-Leray if the $i$ th reduced homology group $\tilde{H}_{i}(\mathrm{~L})$ (over $\mathbb{Q}$ ) vanishes for every induced subcomplex $\mathrm{L} \leq \mathrm{K}$ and every $i \geq d$.

We mention several remarks regarding $d$-collapsible and $d$-Leray complexes. Deeper properties of them are studied in the following sections.

- The fact that $d$-collapsible complexes are $d$-Leray is simple (for a reader familiar with homology) since $d$-collapsing does not affect homology of dimension $d$ or more.

It is a bit less trivial to show that a $d$-representable complex $\mathrm{K}$ is $d$ collapsible. The idea is to slide a generic hyperplane (from infinity to minus infinity) over a $d$-representation for $\mathrm{K}$ and gradually cut off 

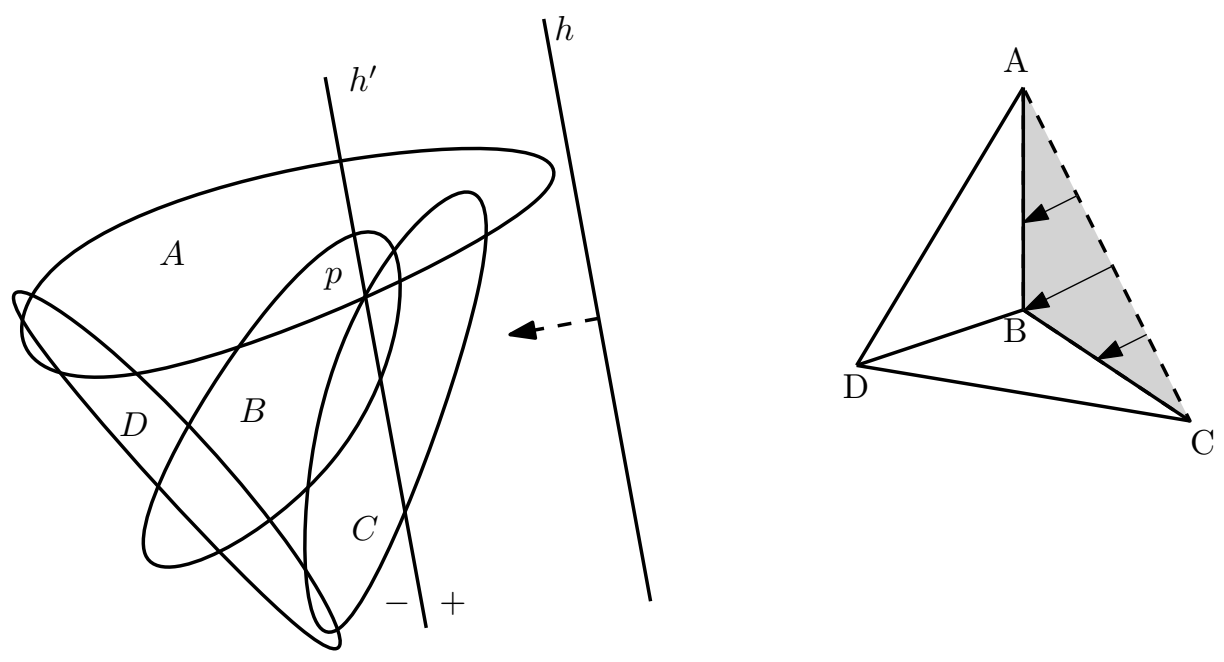

Figure 3: A schematic sketch of the proof of Wegner's theorem. A generic hyperplane $h$ is slided from infinity to minus infinity until there is a nontrivial intersection of the convex sets on its positive side. In this case it slides to $h^{\prime}$ and it cuts off $A \cap B \cap C$ (it also cuts off $A \cap C$, but for the moment we consider a maximal collection). From genericity there is a single point $p \in A \cap B \cap C \cap h^{\prime}$. It can be shown (using Helly's theorem) that there is only at most $d$ sets of the starting collection necessary to obtain $p$. In this case $\{p\}=A \cap C \cap h^{\prime}$. Thus we obtain a $d$-collapse with $\sigma=\{A, C\}$ and $\tau=\{A, B, C\}$. Finally, $A \cap\left(h^{\prime}\right)^{-}, \ldots, D \cap\left(h^{\prime}\right)^{-}$form a $d$-representation for the resulting collapsed complex thus the procedure can be repeated.

whatever is on the positive side of the hyperplane. See Figure 3 and the text bellow the picture for a more detailed sketch. The reader is referred to Weg75 for full details.

The inclusion of $d$-representable complexes in $d$-Leray complexes can be also deduced, without using Wegner's results, from the nerve theorem (see Theorem 5.1).

- A $d$-dimensional simplicial complex is $(d+1)$-collapsible and hence also $(d+1)$-Leray. For a complex $\mathrm{K}$ the smallest possible $\ell$ such that $\mathrm{K}$ is $d$-Leray is traditionally called the Leray number of $\mathrm{K}$.

- Neither $d$-representability, $d$-collapsibility nor the Leray number is an invariant under a homeomorphism: the full simplex $\Delta_{m}$ is 0-represent- 
able; however, its barycentric subdivision is not even $(m-1)$-Leray, since it contains an $(m-1)$-sphere as an induced subcomplex.

- It is not very difficult to see that every induced subcomplex of a $d$ collapsible complex is again $d$-collapsible. If $\mathrm{K}\left[V^{\prime}\right] \leq \mathrm{K}$ and $\mathrm{K} \rightarrow \mathrm{K}_{1} \rightarrow$ $\cdots \rightarrow \emptyset$ is a $d$-collapsing of $\mathrm{K}$, then $\mathrm{K}\left[V^{\prime}\right] \rightarrow \mathrm{K}_{1}\left[V^{\prime}\right] \rightarrow \cdots \rightarrow \emptyset=\emptyset\left[V^{\prime}\right]$ is a $d$-collapsing for $\mathrm{K}\left[V^{\prime}\right]$, where some steps are possibly trivial, i.e., $\mathrm{K}_{i}\left[V^{\prime}\right]=\mathrm{K}_{i+1}\left[V^{\prime}\right]$.

- The Helly theorem easily follows from the fact that $d$-representable complexes are contained in $d$-collapsible ones (or $d$-Leray ones). For we have that a $d$-dimensional simplicial hole is neither $d$-collapsible (nor $d$-Leray).

On the other hand these two notions provide (much) stronger limitations to intersection patterns than the Helly theorem. For instance they also exclude (in dimension 2) the boundary of the octahedron (i.e., the simplicial complex with vertices $\{-3,-2,-1,1,2,3\}$ and faces $\alpha$ such that there is no $i \in\{1,2,3\}$ with $-i, i \in \alpha)$ or a triangulation of a torus.

The gaps among these notions are discussed in more detail in the following section.

\section{Gaps among the notions}

In this section we overview how the notions of $d$-representable, $d$-collapsible and $d$-Leray complexes differ. We also relate these differences with the dimension of the complex.

\subsection{Every finite simplicial complex is $d$-representable for $d$ big enough}

Let $\mathrm{K}$ be a simplicial complex on vertex set $\{1, \ldots, n\}$. Let $x_{1}, \ldots, x_{n}$ be affinely independent points in $\mathbb{R}^{n-1}$ (i.e., they form a simplex). For a nonempty face $\alpha=\left\{a_{1}, \ldots, a_{t}\right\} \in \mathrm{K}$ let $b_{\alpha}$ be the barycentre of the points $x_{a_{1}}, \ldots, x_{a_{t}}$. Then for $i \in\{1, \ldots, n\}$ we set $C_{i}:=\operatorname{conv}\left\{b_{\alpha}: i \in \alpha, \alpha \in \mathrm{K}\right\}$. The reader is welcome to check that sets $C_{i_{1}}, \ldots, C_{i_{k}}$ intersect if and only if $\left\{i_{1}, \ldots, i_{k}\right\} \in \mathrm{K}$. Thus, the nerve of $C_{1}, \ldots, C_{n}$ is isomorphic to K. See Figure 4 for an illustration. (If we really would not care about the dimension, it would be even easier to check the situation where the points $b_{\alpha}$ are set to be the vertices of a simplex of dimension $|K|-1$.) 

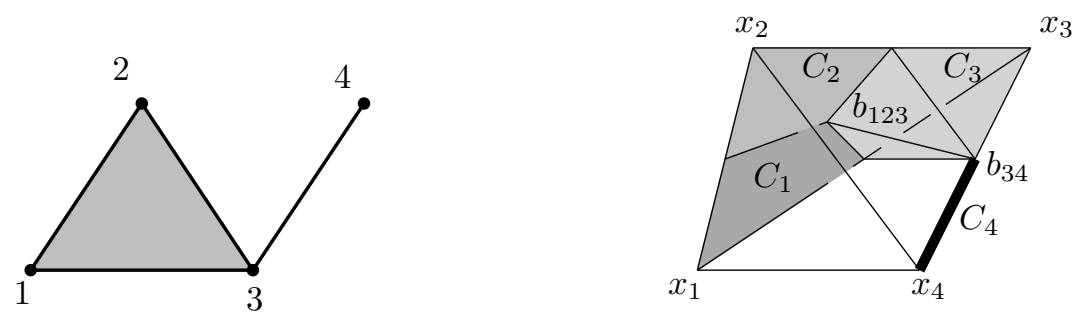

Figure 4: Representing a complex.

There is, however, another way how to obtain a representation of a complex depending on the dimension of the complex.

Theorem 3.1 (Wegner [Weg67], Perel'man [Per85]). Let $\mathrm{K}$ be a d-dimensional simplicial complex. Then $\mathrm{K}$ is $(2 d+1)$-representable.

The value $2 d+1$ in Theorem 3.1 is the least possible. For example, the barycentric subdivisions of the $d$-skeleton of a $(2 d+2)$-dimensional simplex is not $2 d$-representable. Case $d=1$ was established by Wegner Weg67; general case is proved in [Tan11b].

The references for Theorem 3.1 are due to Eckhoff [Eck93]. (Perel'man rediscovered Wegner's result.) Unfortunately, I have not been able to check these sources in detail (the first one is in German, the second one is in Russian). Thus I rather supply an idea of a proof (communicated by Jiří Matoušek).

Sketch of a proof of Theorem 3.1. Let $\mathrm{K}$ be a $d$-representable complex with $n$ vertices.

A $k$-neighborly polytope is a convex polytope such that every $k$ vertices form a face of the polytope. It is well known that there are $2 k$-dimensional $k$-neighborly polytopes with arbitrary number of vertices for every $k \geq 1$. For instance cyclic polytopes satisfy this property. (See, e.g., Mat02 for a background on convex polytopes including cyclic polytopes.)

Let $Q$ be a $(2 d+2)$-dimensional $(d+1)$-neighborly polytope with $n$ vertices. Let $Q^{*}$ be a polytope dual to $Q$. It has $n$ facets and any $d+1$ of its facets share a face of the polytope. Finally, we consider the Schlegel diagram of $Q^{*}$. The Schlegel diagram of an $m$-dimensional convex polytope is a projection of the polytope to $(m-1)$-space through a point beyond one of its facets (the point is very close to the facet). In particular the facets of $Q^{*}$ project to convex sets $C_{1}, \ldots, C_{n}$ in $\mathbb{R}^{2 d+1}$ such that each $d+1$ of them 
share the projection of a face of $Q^{*}$ (on their boundary). Thus if we look at the nerve $\mathrm{N}$ of $C_{1}, \ldots, C_{n}$, then it contains full $d$-skeleton of a simplex with $n$ vertices. Therefore, without loss of generality, we can assume that $\mathrm{K}$ is a subcomplex of $\mathrm{N}$. Let $\vartheta=\left\{C_{i_{1}}, \ldots, C_{i_{j}}\right\}$ be a face of $\mathrm{N}$ which does not belong to $\mathrm{K}$. The sets $C_{i_{1}}, \ldots, C_{i_{j}}$ intersect on their boundaries and it is possible to remove their intersection by removing a small neighborhood of $C_{i_{1}} \cap \cdots \cap C_{i_{j}}$ in each of the sets while keeping the sets convex. Hence only $\vartheta$ and the superfaces of $\vartheta$ disappear from the nerve during this procedure. After repeating the procedure we obtain a collection of convex sets with the nerve $\mathrm{K}$.

\subsection{The gap between representability and collapsibility}

For $d=0$ all three notions 0-representable, 0-collapsible and 0-Leray coincide and they can be replaced with 'being a simplex'.

For $d=1$ : 1-representable complexes are clique complexes over interval graphs; 1-collapsible and 1-Leray complexes are clique complexes over chordal graphs (we remark that results in [LB63, Weg75] easily imply these statements).

For $d \geq 2$ there is perhaps no simple characterization of $d$-representable, $d$-collapsible and $d$-Leray complexes. Wegner Weg75 gave an example of complex, which is 2-collapsible but not 2-representable. Matoušek and the author [MT09] found $d$-collapsible complexes that are not $(2 d-2)$ representable. Later, the author [Tan10b] improved this result by finding 2-collapsible complexes that are not $d$-representable (for any fixed $d$ ). We present some steps of both of the constructions, since even the weaker construction contains some steps of their own interest.

Let $\mathrm{E}$ be a $(d-1)$-dimensional simplicial complex which is not embeddable in $\mathbb{R}^{2 d-2}$. Such a complex always exist, for example the van Kampen

complex $\Delta_{2 d}^{(d-1)}$; see [VK32, or the Flores complex [Flo34], which is the join of $d$ copies of a set of three independent points. The first example is the nerve $\mathrm{N}(\mathrm{E})$. It is $d$-collapsible but not $(2 d-2)$-representable due to the following two propositions [MT09.

Proposition 3.2. Let $\mathrm{K}$ be a simplicial complex such that the nerve $\mathrm{N}(\mathrm{K})$ is n-representable. Then $\mathrm{K}$ embeds in $\mathbb{R}^{n}$, even linearly.

Proposition 3.3. Let $\mathcal{F}$ be a family of sets, each of size at most $n$. Then the nerve $\mathrm{N}(\mathcal{F})$ is n-collapsible. 
The second (stronger) example regards finite projective planes seen as simplicial complexes. Let $(P, \mathcal{L})$ be a finite projective plane, where $P$ is the set of its points and $\mathcal{L}$ is the set of its lines. There is a natural simplicial complex $\mathrm{P}$ associated to the projective plane. Its ground set is $P$ and faces are the collections of points lying on a common line.

It is not hard to show that $P$ is 2-collapsible. Non-representability of $P$ is summarized in the following theorem Tan10b].

Theorem 3.4. For every $d \in \mathbb{N}$ there is a $q_{0}=q_{0}(d)$ such that if a complex $\mathrm{P}$ correspond to projective plane of order $q \geq q_{0}$ then $\mathrm{P}$ is not d-representable.

We also sketch a proof of Theorem 3.4. The reader is referred to the original paper for full details. In contrast to the original paper we present it in an 'inequality form'. For this we need few preliminaries.

One of important ingredients is a selection theorem by Pach [Pac98].

Theorem 3.5. For every positive integer $d$ there is a constant $c=c(d)>0$ with the following property. Let $X \subset \mathbb{R}^{d}$ be a finite set of points in general position. Then there is a point $a \in \mathbb{R}^{d}$ and disjoint disjoint subsets $Z_{1}, \ldots, Z_{d+1}$ of $X$, with $\left|Z_{i}\right| \geq c|X|$ such that the convex hull of every transversal of $\left(Z_{1}, \ldots, Z_{d+1}\right)$ contains a.

We recall that a transversal of a group of sets $\left(Z_{1}, \ldots, Z_{d+1}\right)$ is a set $\left\{z_{1}, \ldots, z_{d+1}\right\}$ such that $z_{i} \in Z_{i}$.

Now we let $\mathcal{S}$ to be a subset of a simplicial complex $\mathrm{K}$ with a vertex set $V$ where $V=\{1, \ldots, n\}$ and $|\mathcal{S}|=s$. For $\mathcal{S}^{\prime} \subset \mathcal{S}$ we define the deficiency of $\mathcal{S}^{\prime}$ as the number of vertices of $\mathrm{K}$ which are not contained in any element of $\mathcal{S}^{\prime}$, i.e., the value $n-\left|\bigcup \mathcal{S}^{\prime}\right|$. A value $\rho(k)$ of a function $\rho:\{1, \ldots, n\} \rightarrow \mathbb{N}$ is defined as the maximum of deficiencies of sets $\mathcal{S}^{\prime} \subseteq \mathcal{S}$ with $k$ elements. Then we have the following inequality.

Proposition 3.6. If $\mathrm{K}$ is d-representable, then

$$
n-(d+1) \rho(c(d) s) \leq \operatorname{dim} \mathrm{K}+1,
$$

where $c(d)$ is the Pach's constant.

Sketch of a proof. Let $C_{1}, \ldots, C_{n}$ be the sets forming the $d$-representation of $\mathrm{K}$, set $C_{i}$ corresponds to a vertex $i$. It can be assumed that these sets are open. For every $\sigma \in \mathcal{S}$ there is a point $x_{\sigma}$ in the intersection of all $C_{i}$ such that $i$ is a vertex of $\sigma$. Let $X=\left\{x_{\sigma}: \sigma \in \mathcal{S}\right\}$. It can be assumed that $X$ is in general position due to the openness of the sets $C_{i}$. So we have 
$Z_{1}, \ldots, Z_{d+1} \subseteq X$ and $a \in \mathbb{R}^{d}$ from Theorem 3.5. For a fixed $j \in\{1, \ldots, d+$ $1\}$ the definition of $\rho$ implies that only $\rho(c(d) s)$ sets among $C_{1}, \ldots, C_{n}$ can avoid the points of the set $Z_{j}$. Thus there is at least $n-(d+1) \rho(c(d) s)$ of the $C_{i}$ that meat all $Z_{j}$, and therefore they contain $a$. Hence the vertices of $\mathrm{K}$ corresponding to these $C_{i}$ form a face of $\mathrm{K}$ of dimension $n-(d+1) \rho(c(d) s)-$ 1.

Theorem 3.4 follows from Proposition 3.6 when $\mathcal{S}$ is set of all maximal simplices of a projective plane P. Then $n=s=q^{2}+q+1, \operatorname{dim} P=q+1$, and $\rho(k) \leq\left(q^{2}+q+1\right)^{3 / 2} / k$ by a theorem of Alon [Alo85, Alo86].

\subsection{The gap between collapsibility and Leray number}

Wegner showed an example of complex which is 2-Leray but not 2-collapsible, namely a triangulation $D$ of the dunce hat. If we consider the multiple join $\mathrm{D} \star \cdots \star \mathrm{D}$ of $d$ copies of $\mathrm{D}$, we obtain a complex which is $2 d$-Leray but not $(3 d-1)$-collapsible. See MT09 for more details.

\section{Algorithmic perspective}

As we consider different criteria for $d$-representability, it is also natural to ask whether there is an algorithm for recognition $d$-representable complexes. We denote this algorithmic question as $d$-REPRESEnTABILITY. More precisely, the input of this question is a simplicial complex. The size of the input is the number of faces of the complex. The value $d$ is considered as a fixed integer. The output of the algorithm is the answer whether the complex is $d$-representable.

We can also ask similar questions for $d$-collapsible and $d$-Leray complexes as relaxations of the previous problem. Thus we have algorithmic problems $d$-COLlapsibility and $d$-LERAYNumBer.

\subsection{Representability}

The first mentioned problem $d$-REPRESENTABILITY is perhaps the most difficult among the three algorithmic questions. It is NP-hard for $d \geq 2$. Reduction can be done in a very similar fashion as a reduction for hardness of recognition intersection graphs of segments [KM89, KM94]. Full details can be found in Tan10a]. On the other hand it is not hard to see that there is a PSPACE algorithm for $d$-REPRESENTABILITY. It is based on solving 
systems of polynomial inequalities. See [KM94, Theorem 1.1(i)(a)] for a very similar reduction.

\subsection{Collapsibility}

It is shown in Tan10a] that $d$-Collapsibility is NP-complete for $d \geq 4$ and it is polynomial time solvable for $d \leq 2$. For $d=3$, the problem remains open.

\subsection{Leray number}

The last question, $d$-LERAYNumBer, is polynomial time solvable. An equivalent characterization of $d$-Leray complexes is when induced subcomplexes are replaced with links of faces (including an empty face). See KM06, Proposition 3.1] for a proof. The tests on links can be done in polynomial time since it is sufficient to test the homology up to the dimension of the complex.

\subsection{Greedy collapsibility}

The algorithmic results above suggest that it is easier to test/compute the Leray number than collapsibility. However, if we are interested in them because of a hint for representability, computing collapsibility still can be more convenient, since $d$-collapsibility is closer to $d$-representability than the Leray number. An example from section 3 is perhaps not so convincing; however, there is a more important example. As it is shown in section 5 , $d$-collapsibility can distinguish collections of convex sets and good covers.

An useful tool for computation could be greedy $d$-collapsibility. We say that a simplicial complex $\mathrm{K}$ is greedily $d$-collapsible if it is $d$-collapsible and any sequence of $d$-collapses of $\mathrm{K}$ ends up in a complex which is still $d$-collapsible. In other words greedy collapsibility allows us to collapse the faces of $\mathrm{K}$ in whatever order without risk of a bad choice. Thus, if a complex is greedily $d$-collapsible, then there is a simple (greedy) algorithm for showing that it is $d$-collapsible. Not all $d$-collapsible complexes are greedily $d$ collapsible. Complexes which are not greedily $d$-collapsible for $d \geq 3$ are constructed in [Tan10a]. However, none of these complexes is $d$-representable. In summary there is a hope for obtaining a simple algorithm for showing that a complex is either $d$-collapsible or it is not $d$-representable if the answer to the following question is true. 
Problem 4.1. Is it true that every d-representable simplicial complex is greedily d-collapsible?

\section{Good covers}

A good cover in $\mathbb{R}^{d}$ is a collection of open sets in $\mathbb{R}^{d}$ such that the intersection of any subcollection is either empty or a contractible (in particular, the sets in the collection are contractible) 1 We consider only finite good covers. A simplicial complex is topologically d-representable if it is isomorphic to the nerve of a (finite) good cover in $\mathbb{R}^{d}$. We should emphasize that (for our purposes) a good cover need not cover whole $\mathbb{R}^{d}$.

Topologically $d$-representable complexes generalize $d$-representable complexes since every collection of convex sets is a good cover.

\section{$5.1 \quad$ Nerve theorems}

Suppose we are given a collection $\mathcal{F}$ of subsets of $\mathbb{R}^{d}$. If the sets are "sufficiently nice" and also all their intersections are sufficiently nice then the nerve of the collection, $\mathrm{N}(\mathcal{F})$, is homotopy equivalent to the union of the sets in the collection, $\cup \mathcal{F}$. For a weaker assumption on "sufficiently nice", it is possible to derive not necessarily homotopy equivalence, but at least equivalence on homology (up to some level). Such results are known as homotopic/homological nerve theorems.

We mention here a one of possible versions (suitable for our purposes); see [Hat01, Corollary 4G.3].

Theorem 5.1 (A homotopy nerve theorem). Let $\mathcal{F}$ be a collection of open contractible sets in a paracompact space $X$ such that $\bigcup \mathcal{F}=X$ and every nonempty intersection of finitely many sets in $\mathcal{F}$ is contractible (or empty). Then the nerve $\mathrm{N}(\mathcal{F})$ and $X$ are homotopy equivalent.

Corollary 5.2. The nerve of every good cover is d-Leray.

\subsection{Good covers versus collections of convex sets}

Good covers have many similar properties as collections of convex sets. Many results on intersection patterns of convex sets can be generalized for

\footnotetext{
${ }^{1}$ The definition of a good cover is not fully standard in the literature. For example, it may be assumed that sets in the collection are closed instead of open, or that the intersections are homeomorphic to (open) balls instead of contractible. These differences are not essential for the most of the purposes mentioned here, because all these options satisfy the assumptions of a nerve theorem (see the text bellow).
} 


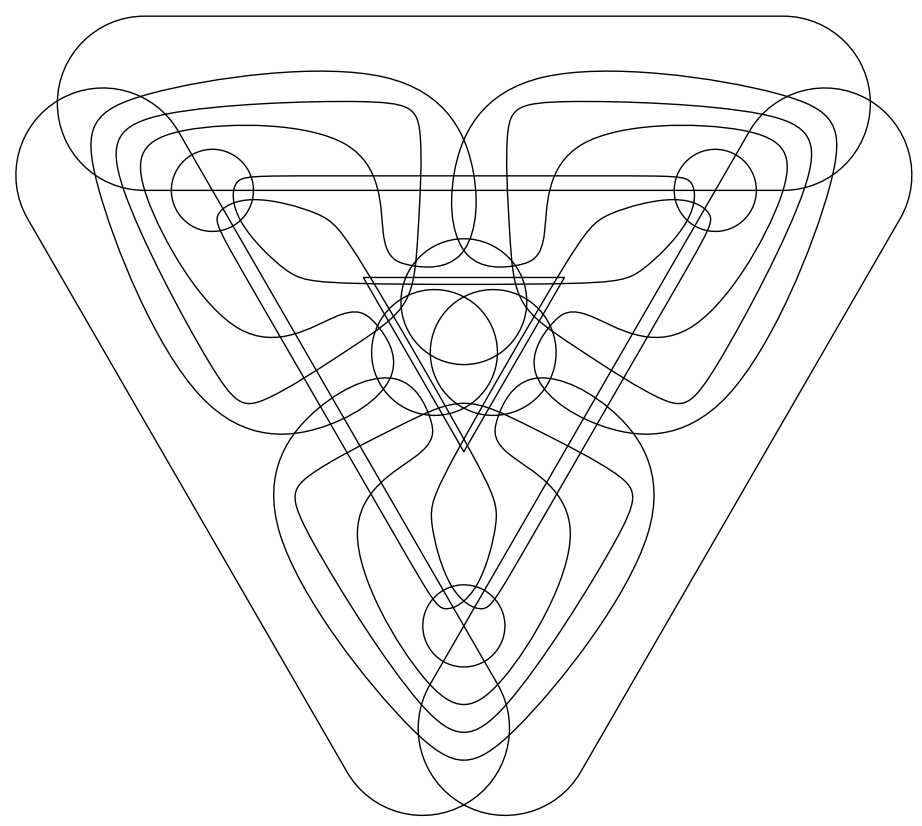

Figure 5: A good cover $\mathcal{F}$ such that the nerve of $\mathcal{F}$ is even not $d$-collapsible.

good covers. We will discuss these generalizations in the following section. An exceptional case is Theorem 3.4 which cannot be generalized for good covers.

On the other hand it is not hard to see that topologically $d$-representable complexes are strictly more general than $d$-representable complexes for $d \geq$ 2. There is a less trivial example on Figure 5 showing that there is a complex which is topologically $d$-representable but not $d$-collapsible. Originally, Wegner conjectured that there is no such example. See [Tan11a for more details.

In addition, this example also distinguishes good covers in $\mathbb{R}^{2}$ and arrangements of convex sets in a topological plane:2 An arrangement of pseudolines is a set of curves (called pseudolines) in the plane such that every two pseudolines intersect in exactly one point. The most convenient way is perhaps to think of the plane as a subset of the real projective plane; then we can allow even "parallel" pseudolines. Such an arrangement can be extended to a topological plane where there is a pseudoline through every pair of points. See, e.g., GPWZ94 for more precise definitions and another

\footnotetext{
${ }^{2}$ This observation is by Xavier Goaoc.
} 
background. A convex set in a topological plane is such a subset that every two points are connected with a "segment" of a pseudoline. The nerve of a bounded collection of convex sets in a topological plane is 2-collapsible. This can be shown in a very similar way as Wegner's theorem on inclusion of $d$-representable complexes in $d$-collapsible ones. Thus the example from Figure 5 cannot be a collection of convex sets in a topological plane.

\section{Helly type theorems}

In this section we overview some Helly-type results on intersection patterns of convex sets. We always start with geometrical formulation. Then we reformulate such a result via $d$-representable simplicial complexes. We also discuss possible extensions to $d$-collapsible or $d$-Leray complexes. (The former ones then have geometric consequences for good covers.)

\subsection{The Helly theorem.}

For completeness of this section we also recall the Helly theorem mentioned in the introduction.

We have the following geometric formulation.

Theorem 6.1 (Helly, Hel23]). If $C_{1}, \ldots, C_{n}$ are convex sets in $\mathbb{R}^{d}, n \geq$ $d+1$, and any collection of $d+1$ sets among $C_{1}, \ldots, C_{n}$ has a nonempty intersection, then all the sets have a common point.

A topological extension of the Helly theorem was proved few years later by Helly himself [Hel30]. His setting was for good covers. We present here a setting for $d$-Leray complexes. Note that the theorem stated here is trivial; however, the fact that it is meaningful relies on Corollary 5.2

Theorem 6.2. The Helly number of a d-Leray simplicial complex is at most $d+1$.

Theorem 6.1 is a consequence of Theorem 6.2 if it is used for $d$-representable complexes.

\subsection{The colorful Helly theorem.}

The colorful Helly theorem regards the situation where convex sets are colored. If there is enough color classes and every rainbow collection of the colored sets contains a point in common, then the sets of a certain color class contain a point in common. 
Theorem 6.3 (Colorful Helly, Lovász [Lov74]). Let $\mathcal{F}_{1}, \ldots, \mathcal{F}_{d+1}$ be families of convex sets in $\mathbb{R}^{d}$. Suppose that for every choice $F_{1} \in \mathcal{F}_{1}, \ldots, F_{d+1} \in \mathcal{F}_{d+1}$ the intersection $F_{1} \cap \cdots \cap F_{d+1}$ is nonempty. Then there is $i \in[d+1]$ such that the intersection of the sets in $\mathcal{F}_{i}$ is nonempty.

The Helly theorem is the consequence of the colorful Helly theorem if we set $\mathcal{F}_{1}=\mathcal{F}_{2}=\cdots=\mathcal{F}_{d+1}$.

The reformulation via simplicial complexes is the following.

Let $V$ be a finite set partitioned into disjoint color classes $V_{1}, \ldots, V_{k}$. A subset $W \subseteq V$ is rainbow if $\left|V_{i} \cap W\right| \leq 1$ for $i \in[k]$.

Theorem 6.4. Let $\mathrm{K}$ be a d-representable simplicial complex with vertices partitioned into $d+1$ color classes. Assume that every rainbow subset of vertices is a simplex of $\mathrm{K}$. Then there is a color class such that its vertices form a simplex of $\mathrm{K}$.

Let $\mathrm{M}^{\prime}$ be a simplicial complex with the vertex set $V$ from above whose faces are the rainbow subsets of $V$. It is not hard to see that $\mathrm{M}^{\prime}$ is a matroidal complex of rank $k$.

Kalai and Meshulam KM05] obtained the following matroidal extension of the colorful Helly theorem.

Theorem 6.5. Let $\mathrm{K}$ be a d-collapsible simplicial complex on $V$ and let $\mathrm{M}$ be a matroidal complex on $V$ with rank function $\rho$ such that $\mathrm{M} \subseteq \mathrm{K}$. Then there is a simplex $\alpha \in \mathrm{K}$ such that $\rho(\alpha)=\rho(\mathrm{M})$ and $\rho(V \backslash \alpha) \leq d$.

Theorem 6.5 indeed generalizes Theorem 6.4. If we set $\mathrm{M}=\mathrm{M}^{\prime}$, then $\alpha$ contains all vertices of a certain color class (since $\rho(V \backslash \alpha) \leq d$ ) and moreover $\alpha$ even contains a vertex of every color class (since $\rho(\alpha)=\rho(\mathbf{M})$ ).

More importantly, Kalai and Meshulam KM05] obtained a topological generalization (with a weaker conclusion, but still more general then that of Theorem 6.4).

Theorem 6.6. Let $\mathrm{K}$ be a d-Leray simplicial complex on $V$ and let $\mathrm{M}$ be a matroidal complex on $V$ with rank function $\rho$ such that $\mathrm{M} \subseteq \mathrm{K}$. Then there is a simplex $\alpha \in \mathrm{K}$ such that $\rho(V \backslash \alpha) \leq d$.

\subsection{The fractional Helly theorem.}

Let $\mathcal{C}$ be again a collection of convex sets in $\mathbb{R}^{d}$ (containing at least $d+1$ sets). The Helly theorem assumes that if every $d+1$-tuple has a nonempty intersection then all the sets have point in common. The fractional Helly 
theorem is designed for a situation when many $d+1$-tuples have a nonempty intersection concluding that many sets of the collection have a point in common.

Theorem 6.7 (Fractional Helly, Katchalski and Liu [KL79]). For every $a \in(0,1]$ and $d \in \mathrm{N}$ there is $b=b(d, a) \in(0,1]$ with the following property. Let $\mathcal{C}$ be a collection of $n$ convex sets in $\mathbb{R}^{d}(n \geq d+1)$. Assume that the number of $(d+1)$-tuples with a nonempty intersection is at least $a\left(\begin{array}{c}n \\ d+1\end{array}\right)$. Then there is a point common to at least bn sets in $\mathcal{C}$.

The largest possible value for $b(d, a)$ is $1-(1-a)^{1 /(d+1)}$ due to Kalai [Kal84] and Eckhoff [Eck85] (i.e., it is even known that $b$ cannot be larger). We remark that the Helly theorem is a special case when setting $a=1$.

There is also a topological extension of the fractional Helly theorem by Alon, Kalai, Matoušek and Meshulam [AKMM02 (with the same bound for $b$ ). They actually prove a bit stronger result (in order to obtain topological $(p, q)$-theorem); however, we prefer to avoid the technical details and so we present the result only in this simpler form.

Theorem 6.8. For every $a \in(0,1]$ and $d \in \mathrm{N}$ there is $b=b(d, a) \in(0,1]$ with the following property. Let $\mathrm{K}$ be a d-Leray complex with $n$ vertices $(n \geq d+1)$. Assume that there are at least $a\left(\begin{array}{c}n \\ d+1\end{array}\right)$ d-faces in $\mathrm{K}$. Then there is a face of size at least bn -1 in $\mathrm{K}$.

The largest possible value for $b(d, a)$ is again $1-(1-a)^{1 /(d+1)}$.

\subsection{The $(p, q)$ theorem.}

Let $p, q$ be integers such that $p \geq q \geq d+1$. A family $\mathcal{F}$ of convex sets in $\mathbb{R}^{d}$ has the $(p, q)$ property if among every $p$ sets of $\mathcal{F}$ some $q$ have a nonempty intersection. The pinning number, $\pi(\mathcal{F})$, of a family $\mathcal{F}$ is the smallest number of points in $\mathbb{R}^{d}$ that intersect all members of $\mathcal{F}$.

Theorem 6.9 $((p, q)$-theorem, Alon and Kleitman AK92]). For every $p \geq$ $q \geq d+1$ there is a number $C=C(p, q, d)$ such that $\pi(\mathcal{F}) \leq C$ for every family of convex sets in $\mathbb{R}^{d}$ with $(p, q)$ property.

The $(p, q)$ theorem was originally conjectured by Hadwiger and Debrunner.

In order not to introduce a new symbol, we let $C$ denote the smallest constant for which is the assertion of the $(p, q)$ theorem valid. The Helly theorem simply says that $C(d+1, d+1, d)=1$. In general, there are, 


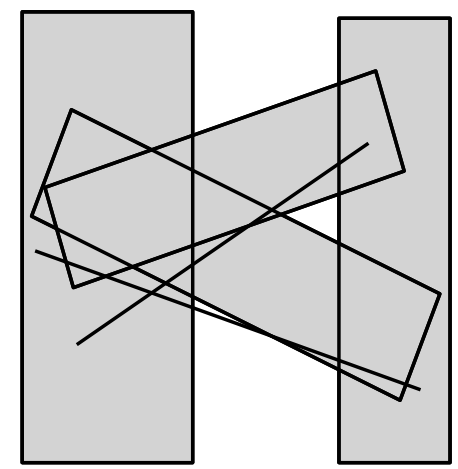

Figure 6: Six convex sets with $(4,3)$ property and pinning number 3.

however, big gaps between lower and upper bounds for $C$. In the first nontrivial case it is relatively easy to come up with an example showing $C(4,3,2) \geq 3$; see Figure 6. Kleitman, Gyárfás and Tóth [KGT01 proved that $C(4,3,2) \leq 13$. The author of this survey believes that the actual value of $C(4,3,2)$ is much closer to 3 then 13 ; however, it seems difficult to obtain the precise value. It is widely open what is the value of $C(p, q, d)$ for larger $p, q$ and $d$.

Now we reformulate the setting for simplicial complexes. A simplicial complex $\mathrm{K}$ has $(p, q)$ property for $p \geq q$ if among every $p$ vertices of $\mathrm{K}$ there is $q$ of them forming a face (of dimension $q-1$ ). The pinning number, $\pi(K)$, of $\mathrm{K}$ is the smallest number of faces of $\mathrm{K}$ such that every vertex of $\mathrm{K}$ is in at least one of these faces. Then the statement of the $(p, q)$ theorem remains valid even for $d$-Leray complexes and consequently for good covers due to Alon et al. AKMM02] 3

Theorem 6.10. For every $p \geq q \geq d+1$ there is a number $C^{\prime}=C^{\prime}(p, q, d)$ such that $\pi(\mathrm{K}) \leq C^{\prime}$ for every d-Leray complex $\mathrm{K}$ with $(p, q)$ property.

\subsection{The Amenta theorem.}

In all previous cases we were considering properties of collections of convex sets. Now we replace convex sets with a finite disjoint union of convex sets. It turns out that there is also a Helly-type theorem for this case (if we keep this property also for intersections).

\footnotetext{
${ }^{3}$ Precisely speaking, Alon et al. state the theorem for good covers only; however, the same reasoning can be used for $d$-Leray complexes.
} 

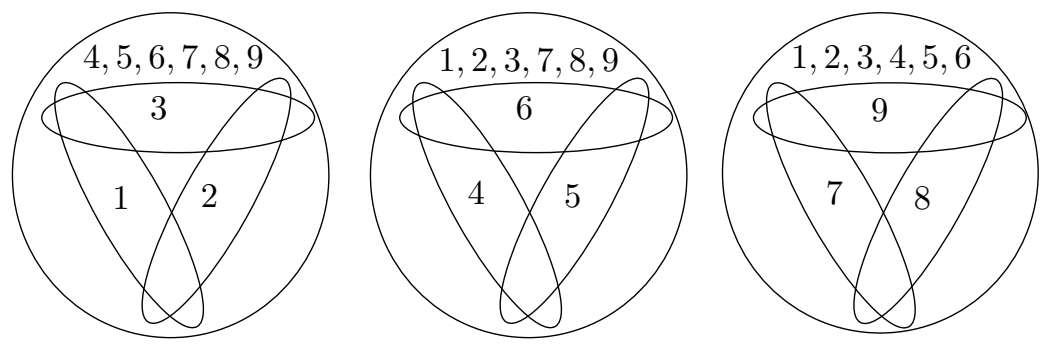

Figure 7: The lower bound for the Amenta's theorem. The convex hulls are a bit enlarged in order to make the figure more lucid. Moreover, the notation of the sets is simplified.

Let $\mathcal{F}$ be a finite family of subsets of some ground set. A family $\mathcal{G}$ is $(\mathcal{F}, k)$-family if for every nonempty $\mathcal{G}^{\prime} \subseteq \mathcal{G}$ the intersection of elements of $\mathcal{G}^{\prime}$ is the disjoint union of at most $k$ members of $\mathcal{F}$. We have defined the Helly number only for simplicial complexes. For purposes of this subsection we say that a family $\mathcal{F}$ has Helly number $h=h(\mathcal{F})$ equal to the Helly number of the nerve of $\mathcal{F}$. (Here we allow $\mathcal{F}$ to be possibly infinite.)

Theorem 6.11 (Amenta Ame96). Let $\mathcal{F}$ be a finite family of compact convex sets in $\mathbb{R}^{d}$. Let $\mathcal{G}$ be an $(\mathcal{F}, k)$-family. Then $h(\mathcal{G}) \leq k(d+1)$.

The bound $k(d+1)$ in Amenta's theorem is optimal as can be shown with the following example. Let $\mathcal{C}=\left\{C_{1}, \ldots, C_{d+1}\right\}$ be a collection of convex sets in $\mathbb{R}^{d}$ such that every $d$ of them has nonempty intersection; however, the intersection of the whole collection is empty (for example, $\mathcal{C}$ might be the collection of facets of a $d$-simplex). Let $\mathcal{C}_{*}=\mathcal{C} \cup\{C\}$ where $C$ is the convex hull of the union of sets in $\mathcal{C}$. Let us consider $k$ disjoint copies $\mathcal{C}_{*}^{i}=\left\{C_{1}^{i}, \ldots, C_{d+1}^{i}, C^{i}\right\}$ of $\mathcal{C}_{*}$ for $i \in\{1, \ldots, k\}$ such that the sets $C^{i}$ are pairwise disjoint. Now we construct sets $D_{j}^{i}$ for $i \in\{1, \ldots, k\}$, $j \in\{1, \ldots, d+1\}$ by setting

$$
D_{j}^{i}=\left(\bigcup_{m \neq i} C^{m}\right) \cup C_{j}^{i} .
$$

Then $\mathcal{G}=\left\{D_{j}^{i}\right\}$ is an $(\mathcal{F}, k)$-family and $h(\mathcal{G})=k(d+1)$. See Figure 7 .

Now we focus on a topological version. Due to the fact that we consider union of sets, there is no simple statement for a topological generalization of Amenta's theorem using $d$-Leray complexes. Thus we prefer set up the statement for good covers in this case. 
Theorem 6.12 (Kalai, Meshulam [KM08]). Let $\mathcal{F}$ be a finite good cover in $\mathbb{R}^{d}$. Let $\mathcal{G}$ be an $(\mathcal{F}, k)$-family. Then $h(\mathcal{G}) \leq k(d+1)$.

Eckhoff and Nischke [EN09 recently proved Amenta's theorem in very abstract setting via Morris's pigeonhole principle. A (possibly infinite) family $\mathcal{F}$ is intersectional, if for any finite subfamily $\mathcal{F}^{\prime}$, the intersection $\cap \mathcal{F}^{\prime}$ is either empty or belongs to $\mathcal{F}$. We call $\mathcal{F}$ nonadditive if for any finite subfamily $\mathcal{F}^{\prime}$ of disjoint sets (at least two nonempty), $\cup \mathcal{F}^{\prime} \notin \mathcal{F}$. The following theorem generalizes the previous two theorems by setting $\mathcal{F}$ to be either a family of convex compact sets, or a family of all intersections of a good cover.

Theorem 6.13. Let $\mathcal{F}$ be an intersectional and nonadditive set family. If $\mathcal{G}$ is an $(\mathcal{F}, k)$ family, then $h(\mathcal{G}) \leq k h(\mathcal{F})$.

From another point of view, a generalization of Theorem 6.12 for collections of sets in more general topological spaces (than $\mathbb{R}^{d}$ ) is obtained by Colin de Verdière, Ginot and Goaoc CGG11. More importantly their generalization applies to collections of sets that need not come from good covers. For instance it applies to a collection of two sets homeomorphic to a ball which intersect in two balls.

By $\Gamma$ we denote a locally arc-wise connected topological space. Then $d_{\Gamma}$ is the smallest integer such that every open subset of $\Gamma$ has a trivial $\mathbb{Q}$-homology in dimension $d_{\Gamma}$ and higher. An open subset of $\Gamma$ with singular $\mathbb{Q}$-homology equivalent to a point is a $\mathbb{Q}$-homology cell. A family of open subsets of $\Gamma$ is acyclic if for every nonempty subfamily $\mathcal{G} \subset \mathcal{F}$, the intersection of $\mathcal{G}$ is a disjoint union of $\mathbb{Q}$-homology cells. Colin de Verdière et al prove the following result.

Theorem 6.14. Let $\mathcal{F}$ be a finite acyclic family of open subsets of locally arc-wise connected topological space $\Gamma$. If any subfamily of $\mathcal{F}$ intersect in at most $k$ connected components, then the Helly number of $\mathcal{F}$ is at most $k\left(d_{\Gamma}+1\right)$.

In particular, $d_{\mathbb{R}^{d}}=d$ and every good cover is acyclic, therefore Theorem 6.14 indeed generalizes Theorem 6.12. Let us also remark that $d_{\Gamma}=d$ for a $d$-dimensional manifold which is either noncompact or nonorientable and $d_{\Gamma}=d+1$ for a compact orientable manifold.

Theorem 6.14 can be furthermore generalized when the homology is zero only from a certain dimension. For this case we already refer the reader to CGG11. On the other, the case when the homology vanish for small dimensions is considered in (preceding) paper [Mat97]. The bound to the Helly number is, however, much weaker. 


\section{Acknowledgement}

I thank Xavier Goaoc for discussions on new generalizations of Amenta's theorem and additional remarks, Jiří Matoušek for many valuable comments to the preliminary version of the survey, and also Janos Pach for discussions about $d$-dimensional complexes which are not $2 d$-representable.

\section{References}

[AK92] N. Alon and D. Kleitman. Piercing convex sets and the Hadwiger Debrunner $(p, q)$-problem. Adv. Math., 96(1):103-112, 1992.

[AKMM02] N. Alon, G. Kalai, J. Matoušek, and R. Meshulam. Transversal numbers for hypergraphs arising in geometry. Adv. in Appl. Math., 130:2509-2514, 2002.

[Alo85] N. Alon. Expanders, sorting in rounds and superconcentrators of limited depth. In Proc. 17th ACM Sympos. on Theory of Comput., pages 98-102, 1985.

[Alo86] N. Alon. Eigenvalues, geometric expanders, sorting in rounds, and Ramsey theory. Combinatorica, 6(3):207-219, 1986.

[Ame96] N. Amenta. A short proof of an interesting Helly-type theorem. Discrete Comput. Geom, 15:423-427, 1996.

[BB97] L. J. Billera and A. Björner. Face numbers of polytopes and complexes. In Handbook of discrete and computational geometry, CRC Press Ser. Discrete Math. Appl., pages 291-310. CRC, Boca Raton, FL, 1997.

[CGG11] É. Colin de Verdière, G. Ginot, and X. Goaoc. Helly numbers of acyclic families. Preprint; http://arxiv.org/abs/1101.6006, 2011.

[Eck85] J. Eckhoff. An upper-bound theorem for families of convex sets. Geom. Dedicata, 19(2):217-227, 1985.

[Eck93] J. Eckhoff. Helly, Radon and Carathéodory type theorems. In P. M. Gruber and J. M. Wills, editors, Handbook of Convex Geometry. North-Holland, Amsterdam, 1993. 
[EN09] J. Eckhoff and K.-P. Nischke. Morris's pigeonhole principle and the Helly theorem for unions of convex sets. Bull. Lond. Math. Soc., 41(4):577-588, 2009.

[Flo34] A. Flores. Über $n$-dimensionale Komplexe die im $R_{2 n+1}$ absolut selbstverschlungen sind. Ergeb. Math. Kolloq., 4:6-7, $1932 / 1934$.

[GPW93] J. E. Goodman, R. Pollack, and R. Wenger. Geometric transversal theory. In New trends in discrete and computational geometry, volume 10 of Algorithms Combin., pages 163-198. Springer, Berlin, 1993.

[GPWZ94] J. E. Goodman, R. Pollack, R. Wenger, and T. Zamfirescu. Arrangements and topological planes. Amer. Math. Monthly, 101(9):866-878, 1994.

[Hat01] A. Hatcher. Algebraic Topology. Cambridge University Press, Cambridge, 2001.

[Hel23] E. Helly. Über mengen konvexer Körper mit gemeinschaftlichen Punkten. Jahresber. Deustch. Math.-Verein., 32:175-176, 1923.

[Hel30] E. Helly. Über Systeme von abgeschlossenen Mengen mit gemeinschaftlichen Punkten. Monaths. Math. und Physik, 37:281-302, 1930 .

[Kal84] G. Kalai. Intersection patterns of convex sets. Israel J. Math., 48(2-3):161-174, 1984.

[Kal02] G. Kalai. Algebraic shifting. In Computational commutative algebra and combinatorics (Osaka, 1999), volume 33 of Adv. Stud. Pure Math., pages 121-163. Math. Soc. Japan, Tokyo, 2002.

[KGT01] D. J. Kleitman, A. Gyárfás, and G. Tóth. Convex sets in the plane with three of every four meeting. Combinatorica, 21(2):221-232, 2001. Paul Erdős and his mathematics (Budapest, 1999).

[KL79] M. Katchalski and A. Liu. A problem of geometry in $R^{n}$. Proc. Amer. Math. Soc., 75:284-288, 1979. 
[KM89] J. Kratochvíl and J. Matoušek. NP-hardness results for intersection graphs. Coment. Math. Univ. Carolin., 30:761-773, 1989.

[KM94] J. Kratochvíl and J. Matoušek. Intersection graphs of segments. J. Comb. Theory Ser. B, 62(2):289-315, 1994.

[KM05] G. Kalai and R. Meshulam. A topological colorful Helly theorem. Adv. Math., 191(2):305-311, 2005.

[KM06] G. Kalai and R. Meshulam. Intersections of Leray complexes and regularity of monomial ideals. J. Combin. Theory Ser. A, 113(7):1586-1592, 2006.

[KM08] G. Kalai and R. Meshulam. Leray numbers of projections and a topological Helly type theorem. J. Topology, 1(3):551-556, 2008.

[LB63] C. G. Lekkerkerker and J. Ch. Boland. Representation of a finite graph by a set of intervals on the real line. Fund. Math., 51:45-64, 1962/1963.

[Lov74] L. Lovász. Problem 206. Matematikai Lapok, 25:181, 1974.

[Mat97] J. Matoušek. A Helly-type theorem for unions of convex sets. Discrete Comput. Geom., 18(1):1-12, 1997.

[Mat02] J. Matoušek. Lectures on Discrete Geometry. Springer-Verlag New York, Inc., 2002.

[Mat03] J. Matoušek. Using the Borsuk-Ulam Theorem. Springer, Berlin etc., 2003.

[MT09] J. Matoušek and M. Tancer. Dimension gaps between representability and collapsibility. Discrete Comput. Geom., 42(4):631-639, 2009.

[Mun84] J. R. Munkres. Elements of Algebraic Topology. Addison Wesley, 1984.

[Pac98] J. Pach. A Tverberg-type result on multicolored simplices. Comput. Geom., 10:71-76, 1998. 
[Per85] G. Ya. Perel'man. Realization of abstract $k$-skeletons of intersections of convex polyhedra in $R^{2 k-1}$. Geometric Questions in the Theory of Functions and Sets (Kalinin. Gos. Univ., Kalinin), pages 129-131, 1985. In Russian.

[Tan10a] M. Tancer. $d$-collapsibility is NP-complete for $d$ greater or equal to 4. Chicago Journal of Theoretical Computer Science, 2010(3):1-28, June 2010.

[Tan10b] M. Tancer. Non-representability of finite projective planes by convex sets. Proc. Amer. Math. Soc., 138(9):3285-3291, 2010.

[Tan11a] M. Tancer. A counterexample to Wegner's conjecture on good covers. Discrete $\&$ Computational Geometry, pages 1-9, 2011. Online-first.

[Tan11b] M. Tancer. $d$-representability of simplicial complexes of fixed dimension. Preprint; http://arxiv.org/abs/1107.1170, 2011. To appear in Journal of Computational Geometry.

[vK32] R. E. van Kampen. Komplexe in euklidischen Räumen. Abh. Math. Sem. Hamburg, 9:72-78, 1932. Berichtigung dazu, ibid. (1932) 152-153.

[Weg67] G. Wegner. Eigenschaften der Nerven homologisch-einfacher Familien im $R^{n}$. PhD thesis, Universität Göttingen, 1967. In German.

[Weg75] G. Wegner. $d$-collapsing and nerves of families of convex sets. Arch. Math., 26:317-321, 1975. 